\title{
How to build a 3D extinction model of the Galaxy
}

\author{
J. R. D. Lépine ${ }^{1}$ \& E. B. Amôres ${ }^{1}$ \\ ${ }^{1}$ Instituto de Astronomia, Geofísica e Ciências Atmosféricas, Universidade de São Paulo, \\ Cidade Universitária, São Paulo, SP, Brazil; email: jacques@astro.iag.usp.br
}

\begin{abstract}
We show that anomalous extinction (deviations from the traditionally adopted $R_{V}=$ $A_{V} / E(B-V)=3.1$ introduces large uncertainties in the distances of stars, for distances larger than 1-2 kpc. We argue that for such distances and for directions close to the galactic plane, the use of extinction models based on the gas distribution in the Galaxy is safer, for the moment, than the use of extinction maps.
\end{abstract}

Keywords. interstellar extinction, galactic structure

\section{Introduction}

It is usually believed that all that we need to derive the photometric distance of a star, taking into account extinction, is the color excess $E(B-V)$. In reality the existence of anomalous extinction seriously challenges this belief. Besides this, there are two opposite views concerning the possibility of corrections for interstellar extinction based on models. One is that the dust distribution is clumpy and random. Consequently, it does not make any sense to produce models; only empirical extinction maps or tables are useful. The other idea is that the proportionality between gas and dust column densities is well established. Simple models of the gas distribution can be constructed, based on HI and CO surveys, so that extinction is predictable to some extent. Amôres \& Lépine (2005) made an extensive comparison of an extinction model based on the gas distribution with a large sample of stars with known extinctions (the sample of Neckel \& Klare 1980). The comparison proved that the extinction is largely predictable. Interestingly, Neckel \& Klare themselves produced extinction maps based on their sample of stars, which constitutes an opportunity for a comparison between the two approaches.

\section{The problem of anomalous extinction, and discussion}

Neckel \& Klare (1980) in a famous work, determined the spectral type and color excess for more than $7000 \mathrm{O}$ and B stars situated near the galactic plane. They plotted the interstellar extinction $A_{V}$ as a function of distance for many directions, and obtained some unexpected results. In many cases, one can observe in those plots a low extinction up to a distance of the order of $1 \mathrm{kpc}$ (see eg. the direction $l=128, b=0$ ), followed by a step in the extinction up to $\mathrm{A}_{V}=2-3$, and then $A_{V}$ remains constant up to about $5 \mathrm{kpc}$, the maximum distance investigated. It is not surprising to see steps in $A_{V}$, as they are explained by the presence of dense clouds along the line-of-sight. What is surprising is that there are paths of many kpc without steps, as if all the clouds were close to the Sun. Another surprising result, noted by Neckel \& Klare, is that the clouds situated close to the Sun are bigger than the more distant ones.

The explanation for the two unexpected results is that the distances of the more distant stars (and clouds) are overestimated. The clouds contain dense cores in which $R_{V}$ 


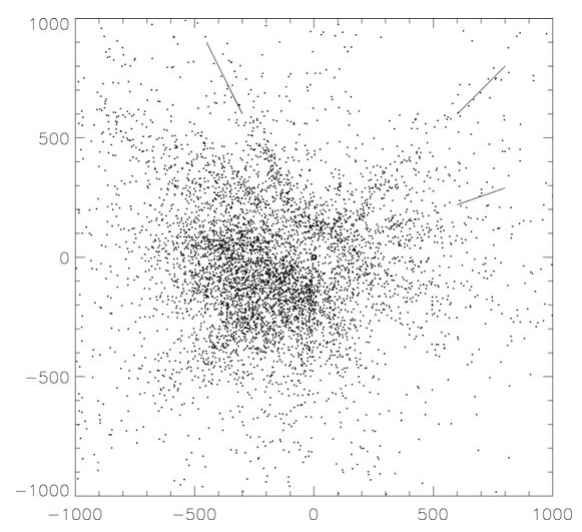

Figure 1. OB stars in the plane of the Galaxy. The distances from the Sun are indicated in parsecs, the centre of the Galaxy is outside the figure, at $(0,-7500)$. The line segments point to examples of directions of alignments of stars or "fingers of God" caused by anomalous extinction.

can be much larger than the classical value 3.1. As a consequence, the extinction is underestimated and the distances are overestimated. The existence of anomalous extinction is not a new result, we only call attention to the fact that the anomalous extinction is so widespread that it affects strongly our understanding of the local structures.

We present in Figure 1 the photometric distances of the sample of OB stars of the solar neighborhood taken from the Hipparcos catalog. We first computed the distances based on the absolute magnitudes expected from their spectral class, with the extinction estimated using $R_{V}=3.1$. We also computed the distances based on the infrared $\mathrm{H}$ magnitudes taken from 2Mass and the relation $A_{H}=0.18 E(V-H)$ derived from the extinction relations by Koornneef 1983. The distances using $\mathrm{V}$ and $\mathrm{H}$ bands are not different, the ones based on $\mathrm{H}$ magnitudes are shown in Figure 1. Since the extinction in band $\mathrm{H}$ is smaller than in $\mathrm{V}$, we would expect that the anomalous extinction would also be smaller. However, it can be seen in the figure that elongated structures like fingers of God are present. The elongated structures cannot be the result of any kind of calibration errors. They are the result of an unpredictable scattering in the values of $R_{V}$. The distance of the first interstellar cloud found along a line-of sight is correct, since the stars are still unaffected by extinction, but the next clouds have distances overestimated. Extinction maps, to be useful for a 3D description of the extinction in the Galaxy, would have to give the distances of all the steps in $A_{V}$, which is not possible at the moment.

How to correct for anomalous extinction? Of course when the data from Gaia become available we will be able to place real distances to the molecular clouds which are responsible for steps in the extinction, up to much larger distances. Meanwhile, it is possible that the approach of Fitzpatrick \& Massa (2009) which describes $R_{V}$ in terms of two color indices could be a good solution.

\section{Acknowledgements}

We thank Leticia Vaz and Nayara Torres for the compilation of the catalog of OB stars.

\section{References}

Amôres, E. B. \& Lépine, J. R. D. 2005, AJ 130, 659

Fitzpatrick, E. L. \& Massa, D., 2009, ApJ 699, 1209

Koornneef, J. 1983, A\& $A$ 128, 84

Neckel, Th. \& Klare, G. 1980, A\&AS 42, 251 